\title{
Effect of Tongxinluo on Nephrin Expression via Inhibition of Notch1/Snail Pathway in Diabetic Rats
}

\author{
Fangqiang Cui, ${ }^{1}$ Dawei Zou, ${ }^{1}$ Yanbin Gao, ${ }^{1}$ Na Zhang, ${ }^{1}$ Jinyang Wang, ${ }^{2}$ Liping Xu, \\ Jianguo Geng, ${ }^{1}$ Jiaoyang $\mathrm{Li}^{1}{ }^{1}$ Shengnan Zhou, ${ }^{1}$ and Xinyao Wang ${ }^{1}$ \\ ${ }^{1}$ Beijing Key Lab of TCM Collateral Disease Theory Research, School of Traditional Chinese Medicine, Capital Medical University, \\ No. 10, Youanmenwai, Xitoutiao, Fengtai District, Beijing 100069, China \\ ${ }^{2}$ Department of Endocrinology, Gansu Provincial People’s Hospital, 204 West Donggang Road, Lanzhou, Gansu 730000, China
}

Correspondence should be addressed to Yanbin Gao; gaoyb111@hotmail.com

Received 3 June 2015; Revised 12 August 2015; Accepted 12 August 2015

Academic Editor: Yuewen Gong

Copyright (C) 2015 Fangqiang Cui et al. This is an open access article distributed under the Creative Commons Attribution License, which permits unrestricted use, distribution, and reproduction in any medium, provided the original work is properly cited.

\begin{abstract}
Podocyte injury is an important mechanism of diabetic nephropathy (DN). Accumulating evidence suggests that nephrin expression is decreased in podocyte in DN. Moreover, it has been demonstrated that tongxinluo (TXL) can ameliorate renal structure disruption and dysfunction in DN. However, the effect of TXL on podocyte injury in DN and its molecular mechanism is unclear. In order to explore the effect of TXL on podocyte injury and its molecular mechanism in DN, our in vivo and in vitro studies were performed. Our results showed that TXL increased nephrin expression in diabetic rats and in high glucose cultured podocyte. Meanwhile, TXL decreased ICN1 (the intracellular domain of notch), HES1, and snail expression in podocyte in vivo and in vitro. More importantly, we found that TXL protected podocyte from injury in DN. The results demonstrated that TXL inhibited the activation of notch1/snail pathway and increased nephrin expression, which may be a mechanism of protecting effect on podocyte injury in DN.
\end{abstract}

\section{Introduction}

Diabetic nephropathy (DN) has become the leading cause of end stage renal disease. As we know, microalbuminuria is an early clinical hallmark of DN. The presence of albuminuria indicates the injury of the glomerular filtration barrier (GFB), of which podocyte is an important component [1]. Accumulate evidence has shown that podocyte loss and injury are an important mechanism of DN [2-4].

Nephrin is a signature molecule of podocyte [5]. Nephrin plays an important role in regulating many pivotal functions of podocyte, including signal transduction and cytoskeletal reorganization. Decreased expression of nephrin is a good marker of podocyte injury. It has been demonstrated that nephrin expression is significantly decreased in DN [6-9].

Evidence is available that notch pathway is a common pathway of podocyte injury [10]. Waters et al. [11] demonstrate that notch pathway activation can lead to podocyte injury in vivo and in vitro. It has been demonstrated that the activation of notch pathway can downregulate nephrin expression in previous studies [11, 12]. Moreover, Gagliardini et al. demonstrate that the notchl and snail signaling is persistently activated in podocyte in $\mathrm{DN}$, which is a major molecular mechanism of decreased nephrin expression [13]. Thus, increased ICN1 and snail expression of podocyte can decrease nephrin expression and then induce podocyte injury in DN. The regulation of ICN1 and snail expression has been an important target for protecting podocyte against injury in DN.

TXL, which is a new kind of Chinese herbal compound, consists of a group of herbal medicines, such as Panax ginseng and Paeonia lactiflora Pallas. TXL has been widely used in clinical practice in China over recent years. It has been demonstrated that TXL is beneficial for heart disease and cerebral disease $[14,15]$. Many previous studies have been performed to explore the mechanism of TXL on heart disease and cerebral disease. It has been found that TXL can protect against pressure overload-induced 
heart failure by activating VEGF/Akt/eNOS pathway [16]. Moreover, Cui et al. have demonstrated that TXL protects cardiac microvascular endothelial cells through increasing autophagy and activating MEK/ERK pathway [17]. Liu et al. found that TXL regulates extensive blood-brain barrier disruption in ischemic stroke via its anti-inflammatory effect [18]. Moreover, positive therapeutic efficacy of TXL in patients with $\mathrm{DN}$ was observed in our clinical practice. It has been demonstrated that ginsenoside Rg1, a major active component of TXL, can protect podocyte from injury [19, 20]. More importantly, our previous study has found that TXL can ameliorate renal structure and function through inhibiting renal tubular epithelial-to-mesenchymal transition in diabetic rats [21]. However, the effect of TXL on podocyte injury and its mechanism is unclear.

In the present study, TXL decreased ICN1, HES1, and snail expression and increased nephrin expression in vivo and in vitro. More importantly, TXL had protecting effect on podocyte injury in diabetic rats and in high glucose cultured podocyte. Taken together, our results showed that TXL inhibited the activation of notch1/snail pathway and increased nephrin expression, which may be a mechanism of protecting effect on podocyte injury.

\section{Materials and Methods}

2.1. Preparation of TXL. TXL superfine powder was provided by Shijiazhuang Yiling pharmaceutical Co. (Hebei, China). The herbal drugs of TXL were seriously authenticated and standardized through their marker compounds according to the Chinese Pharmacopoeia (2005). The species, origin, harvest time, medicinal parts, and concoction methods for each component were strictly standardized. The components of TXL were ground to superfine powder with a diameter $\leqslant 10 \mu \mathrm{m}$ by a Micronizer. Moreover, the security of TXL has been approved by the State Food and Drug Administration in China. In our in vivo study, TXL superfine powder was dissolved in aquadistillate and intragastrically administered at $60 \mathrm{mg} / \mathrm{kg}$ for DN rats each day. In our in vitro study, the TXL superfine powder was dissolved in serum-free DMEM/low glucose medium and sonicated for 1 hour. Then the TXL solution was centrifuged at 3,500 rpm for $10 \mathrm{~min}$ and the supernatant was filtrated by a $0.22-\mu \mathrm{m}$ micropore filter. In order to calculate practical volume of dissolved TXL superfine powder, the precipitate was heated and dried. The final concentration of TXL superfine powder solution is $2000 \mu \mathrm{g} / \mathrm{mL}$ [22]. In our in vitro study, the TXL superfine powder solution was diluted by DMEM/low glucose medium for subsequent use.

2.2. Animals. The experiments were in accordance with the National Institutes of Health Guide for the Care and Use of Laboratory Animals and were approved by the Institutional Animal Care and Use Committee at Capital Medical University. Male Sprague-Dawley rats, aged 8 weeks and weighing 180-200 g, were purchased from the Chinese Academy of Medical Sciences (Beijing, China). All rats were maintained in a conventional environment with a regular $12 \mathrm{~h}$ light/dark cycle and $24 \pm 1^{\circ} \mathrm{C}$ temperature. The diabetic rats were induced by intraperitoneally injecting STZ at $60 \mathrm{mg} / \mathrm{kg}$. A total of 12 rats were injected with an equal volume of vehicle ( $0.1 \mathrm{M}$ citrate buffer, $\mathrm{pH} 4.5)$ as control. Forty-eight hours after injection, tail vein blood was collected and serum glucose was detected in all rats. The diabetic rat model was considered to be successful when its serum glucose $\geq 16.7 \mathrm{mmol} / \mathrm{L}$. The diabetic rats were randomly divided into two groups: diabetic nephropathy group (DN group, $n=$ 12) and DN with TXL group (TXL group, $n=12$ ). The rats injected with vehicle were as normal control group (NC group, $n=12)$. The rats of TXL group were treated with TXL solution (TXL superfine powder, $0.75 \mathrm{~g} \cdot \mathrm{kg}^{-1} \cdot \mathrm{d}^{-1}$, gavage) and the rats of NC group and DN group were treated with an equal volume of vehicle (normal saline, NS, gavage). All rats were provided with free access to food and water throughout the experiment. The rats of the three groups were observed for 12 weeks. At the end of $0,4,8$, and 12 weeks, serum glucose and urinary albumin excretion (UAE) were detected. At the end of 12 weeks after STZ, all rats were sacrificed and renal cortex was collected for electron microscope observation and purposed experiments.

2.3. Cell Culture. The conditionally immortalized mouse podocyte line was obtained from the National Platform of Experimental Cell Resources for Sci-Tech. Cell was cultured under permissive conditions at $33^{\circ} \mathrm{C}$ in DMEM/low glucose (Hyclone) medium supplemented with $10 \%$ fetal bovine serum (Excell) and recombinant IFN- $\gamma$ (PEPROTECH) for proliferation. In order to induce differentiation, podocytes were grown under nonpermissive conditions at $37^{\circ} \mathrm{C}$ in DMEM/low glucose medium without recombinant IFN- $\gamma$. When they grew to about $80 \%$ confluence, podocytes were maintained in serum-free conditions for $24 \mathrm{~h}$. To detect the optimum concentration of TXL, podocytes were treated with different concentrations of TXL $(10,25,50,100$, and $150 \mu \mathrm{g} / \mathrm{mL}$ ) for $24 \mathrm{~h}$. The podocytes were divided into three groups: normal control group (NC group, DMEM containing $5.5 \mathrm{mmol} / \mathrm{L}$ glucose $+24.5 \mathrm{mmol} / \mathrm{L}$ mannitol), high glucose group (HG group, DMEM containing $5.5 \mathrm{mmol} / \mathrm{L}$ glucose + $24.5 \mathrm{mmol} / \mathrm{L}$ glucose), and TXL group (DMEM containing $30 \mathrm{mmol} / \mathrm{L}$ glucose $+100 \mu \mathrm{g} / \mathrm{mL}$ ). The podocytes of three groups were treated for $24 \mathrm{~h}$ and then were used for purposed experiments.

MTT Assay. Cells were cultured in a 96-well plate at 5000 cells/well. After incubation with serum-free DMEM, podocytes were treated with different concentrations of TXL $(10,25,50,100,150$, and $200 \mu \mathrm{g} / \mathrm{mL})$ for $24 \mathrm{~h}$. After that, cells were cultured with MTT solution at a concentration of $0.5 \mathrm{mg} / \mathrm{mL}$ for $4 \mathrm{~h}$. The MTT solution was removed and DMSO was added to dissolve the purple crystals of formazan. The optical density (OD) was detected by a spectrophotometer at $570 \mathrm{~nm}$.

2.4. Electron Microscopy. Renal cortexes were fixed with $2 \%$ glutaraldehyde in $0.1 \mathrm{~mol} / \mathrm{L}$ phosphate buffer at $4^{\circ} \mathrm{C}$ for $120 \mathrm{~min}$. They were then sectioned into ultrathin slices and were double stained with $4 \%$ uranyl acetate and lead citrate. The ultrathin slices were observed by a Hitachi 7100 
transmission electron microscope (Hitachi High Technologies, Tokyo, Japan).

2.5. Western Blot Analysis. Renal cortexes and treated cells were collected and lysed as described previously [23]. Equal amounts of protein $(20 \mu \mathrm{g}$ per lane) were separated by electrophoresis through $10 \%$ SDS-PAGE and then transferred to polyvinylidene difluoride membranes. After blocking with $5 \%$ nonfat dry milk in PBS $+0.05 \%$ Tween 20 , the membranes were treated with primary antibody, washed, and then incubated with peroxidase secondary antibody. Antibodies and dilutions included the following: rabbit monoclonal to nephrin antibody $(1: 2000$, Sigma), goat polyclonal to snail antibody (1:500, Everest Biotech), rabbit polyclonal to notchl-cleaved-val1744 antibody (1:2000 Abcam), and mice monoclonal to GAPDH antibody $(1: 20000)$. The blots were visualized with LumiGLO reagent and peroxide, followed by exposure to X-ray film. Western blot analyses were performed at least in triplicate.

2.6. Immunohistochemistry. Renal tissue sections $(4 \mu \mathrm{m})$ were used to perform immunohistochemical staining for nephrin and snail. After antigen retrieval, the sections were incubated with the primary antibodies of nephrin (Abcam, $1: 100)$ and snail (Everest, 1:50). Then renal tissues were incubated with species-specific secondary antibodies and diaminobenzidine. After that, the sections were counterstained with hematoxylin and observed with fluorescent microscope. The semiquantitative analyses for immunohistochemistry study were performed using Image-Pro Plus 6.0 software. Twenty high-power microscope fields of each group were randomly selected, and their optical density (OD) was detected. Mean optical density (MOD) of each group was calculated and then used for statistical analysis.

2.7. Immunofluorescence. Cells were cultured on the cover glasses in 24 -well plates. When grown to about $80 \%$ confluence, cells were fixed with $4 \%$ paraformaldehyde for $30 \mathrm{~min}$. After blocking the nonspecific binding sites, cells were incubated with primary antibodies at $4^{\circ} \mathrm{C}$ overnight. After washing in PBS, cells were incubated with secondary antibodies at room temperature for $2 \mathrm{~h}$, followed by counterstaining with DAPI. Cells were observed under confocal microscope (Leica TCS SP5 MP, Leica, Heidelberg, Germany). Antibodies and dilutions were as follows: rabbit polyclonal to notchl-cleavedvall744 antibody $(1: 200, \mathrm{Abcam})$ and goat polyclonal to snail antibody (1:200, Everest Biotech).

2.8. Hoechst 33258 Staining. Cells were cultured on the cover glasses in 24 -well plates. When grown to about $80 \%$ confluence, cells were fixed with $4 \%$ paraformaldehyde for 30 min. After that, cells were incubated with Hoechst 33258 for $30 \mathrm{~min}$ at room temperature. Cells were observed under fluorescent microscope. Apoptotic cells were identified by nuclear condensation and/or fragmentation. The number of apoptotic cells was counted in 10 random fields per group.

2.9. Phalloidin Staining. Cells were cultured on the cover glasses in 24-well plates. When grown to about $80 \%$ confluence, cells were fixed with $4 \%$ paraformaldehyde for $30 \mathrm{~min}$. After that, cells were incubated with phalloidin for $30 \mathrm{~min}$ at room temperature. After washing in PBS, cells were counterstained with DAPI. Cells were observed under a confocal microscope (Leica TCS SP5 MP, Leica, Heidelberg, Germany).

2.10. Real-Time RT-PCR. Total RNA was isolated using the TRIzol reagent (Invitrogen) according to the manufacturer's instructions. Then RNA was reverse transcribed into cDNAs by the SuperScript RT kit (Invitrogen). Relative mRNA levels were examined using SYBR Green real-time quantitative reverse transcription-PCR (qRT-PCR) (Applied Biosystems) and were calculated by the $2^{-\Delta \Delta \mathrm{Ct}}$ method. The sequences of primers are the following: mice nephrin: forward primer, $5^{\prime}$-CCCAACACTGGAAGAGGTGT- ${ }^{\prime}$, reverse primer, $5^{\prime}$ CTGGTCGTAGATTCCCCTTG-3'; rat HES1: forward primer, $5^{\prime}$-CAAACCAAAGACAGCCTCTG- ${ }^{\prime}$, reverse primer, $5^{\prime}$-ATGCCGGGAGCTATC-TTTCT-3'; mice HES1: forward primer, $5^{\prime}$-CACGACACCGGACAAACCA- $3^{\prime}$, reverse primer, $5^{\prime}$-GCCGGGAGCTATCTTTCTTAAGTG-3'; mice snail: forward primer, $5^{\prime}$-AGCCCAACTATAGCGAGCTG- ${ }^{\prime}$, reverse primer, $5^{\prime}$-CCAGGAGAGA-GTCCCAGATG- $3^{\prime}$. All RT-PCRs were performed in triplicate and the data were presented as mean \pm SD.

2.11. Statistical Analysis. Data were presented as mean \pm SEM. Statistical analyses were performed by one-way ANOVA followed by the Bonferroni multiple comparison test (for comparison of more than 2 groups) or Student's $t$-test (for comparison of 2 groups). $P<0.05$ was considered statistically significant.

\section{Results}

3.1. The Cytotoxicity and Optimum Concentration of TXL for Cultured Podocyte. To detect the cytotoxicity of TXL on podocyte, MTT assay was performed in our study. Our results showed that, compared with control, TXL at 10, 25, 50,100 , and $150 \mu \mathrm{g} / \mathrm{mL}$ has no cytotoxicity on podocyte. However, cell viability was significantly decreased when it was incubated with TXL at $200 \mu \mathrm{g} / \mathrm{mL}$. To determine the optimum concentration of TXL, nephrin protein expression was detected by western blot in cultured podocyte treated with different concentrations of TXL $(10,25,50,100$, and $150 \mu \mathrm{g} / \mathrm{mL}$ ). Our results showed that HG repressed nephrin expression and TXL increased nephrin expression in high glucose cultured podocyte. More importantly, we found that TXL at $100 \mu \mathrm{g} / \mathrm{mL}$ had the optimum effect on nephrin expression in high glucose cultured podocyte (Figure 1).

3.2. TXL Increased Nephrin Expression In Vivo and In Vitro. In our in vivo study, nephrin protein expression was detected by western blot and immunohistochemistry. Compared with NC group, nephrin expression was decreased in diabetic rats. TXL attenuated decreased nephrin expression in diabetic rats. Meanwhile, nephrin mRNA and protein expression were detected in cultured podocyte by RT-PCR and western blot. In accordance with our in vivo study, nephrin protein 


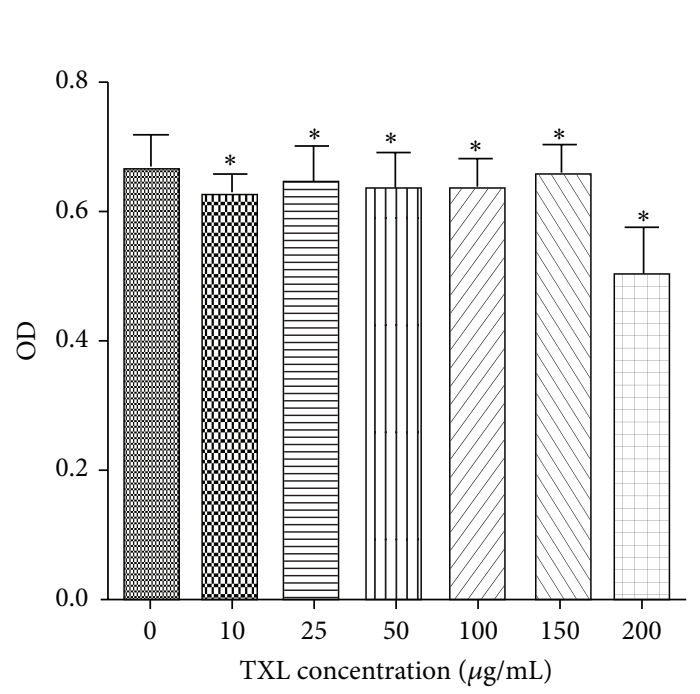

(a)
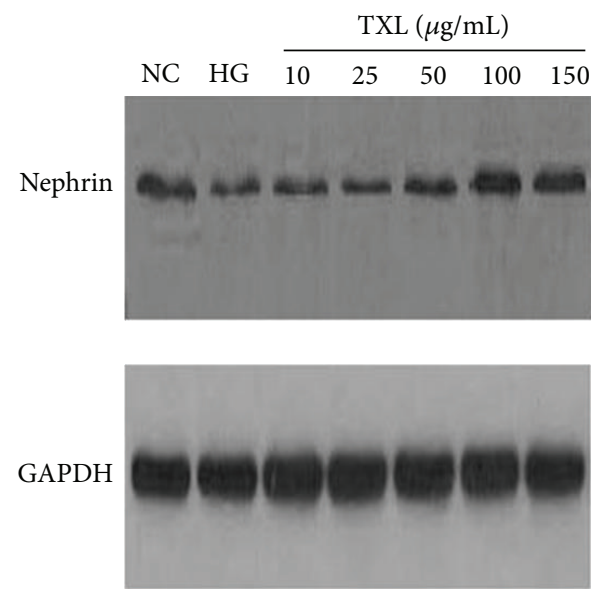

(b)

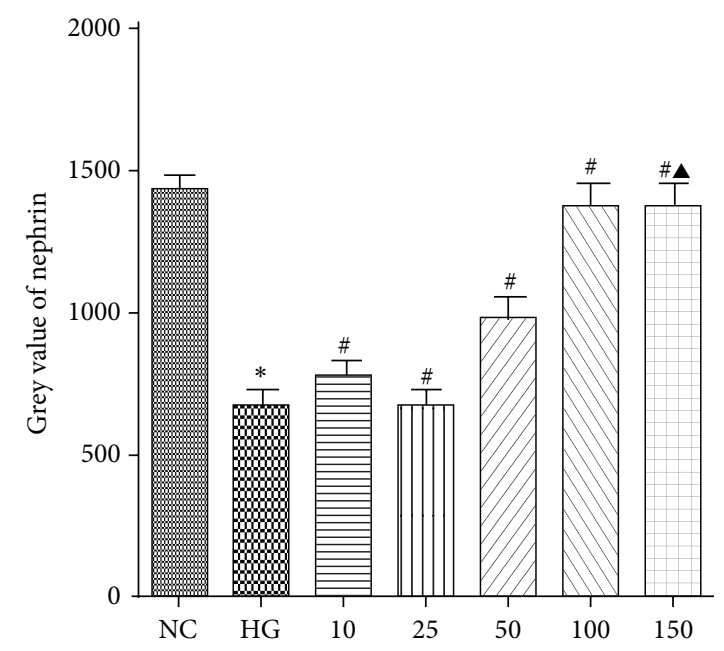

(c)

FIGURE 1: The cytotoxicity and optimum concentration of TXL for cultured podocyte. (a) Comparison of OD in cultured podocyte. The result showed that, compared with control, the OD had not significantly changed with TXL at the concentrations of $10,25,50,100$, and $150 \mu \mathrm{g} / \mathrm{mL}(P>0.05)$; the OD was significantly decreased with TXL at the concentration of $200 \mu \mathrm{g} / \mathrm{mL}(P<0.05)$. (b) Representative band of nephrin protein by western blot in cultured podocyte. (c) Comparison of the grey value of nephrin protein in cultured podocyte $(n=3)$. The result showed that nephrin protein was statistically significantly decreased in HG group compared with NC group $(P<0.05)$. TXL at the concentrations of $10 \mu \mathrm{g} / \mathrm{mL}$ and $25 \mu \mathrm{g} / \mathrm{mL}$ had no effect on nephrin expression compared with HG group $(P>0.05)$. Nephrin expression began to be increased in high glucose cultured podocyte when TXL concentration was added to $50 \mu \mathrm{g} / \mathrm{mL}(P<0.05)$. Nephrin expression was peaked at TXL concentration of $100 \mu \mathrm{g} / \mathrm{mL}(P<0.05)$. When TXL concentration was added to $150 \mu \mathrm{g} / \mathrm{mL}$, nephrin expression had no change compared with the group treated with $100 \mu \mathrm{g} / \mathrm{mL}(P>0.05) .{ }^{*} P<0.05$ versus NG. ${ }^{\#} P<0.05$ versus $\mathrm{HG}$. ${ }^{\wedge} P<0.05$ versus TXL $(100 \mu \mathrm{g} / \mathrm{mL})$.

expression was significantly decreased in HG group compared with NC group $(P<0.05)$. Moreover, nephrin mRNA transcript level was also significantly decreased in high glucose cultured podocyte $(P<0.05)$. TXL reversed decreased nephrin protein expression and mRNA transcript level induced by high glucose $(P<0.05)$ (Figure 2).

3.3. TXL Inhibited the Activation of Notch Pathway In Vivo and In Vitro. As we know, the initial step in the activation of notch pathway is the proteolytic cleaving of the intracellular domain of notch1 by $\gamma$-secretase, with consequent release of ICN1 that translocates into the nucleus. HES1, a downstream target gene of ICN1, is an important component of notch pathway
[24]. Herein, ICN1 and HES1 expression were detected in our in vivo and in vitro study. In our in vivo study, ICN1 and HES1 expression were detected by western blot and RT-PCR, respectively. Our results showed that ICN1 protein expression was increased in DN group. HES1 expression was increased in DN group compared with NC group. TXL decreased ICN1 and HES1 expression in diabetic rats. In our in vitro study, ICN1 expression was detected by immunofluorescence and western blot, and HES1 transcript level was detected by RTPCR. Our immunofluorescence results showed that ICN1 was weakly distributed in the cytoplasm of podocyte in NC group. High glucose increased ICN1 expression and promoted ICN1 translocation from cytoplasm to nucleus. TXL inhibited 


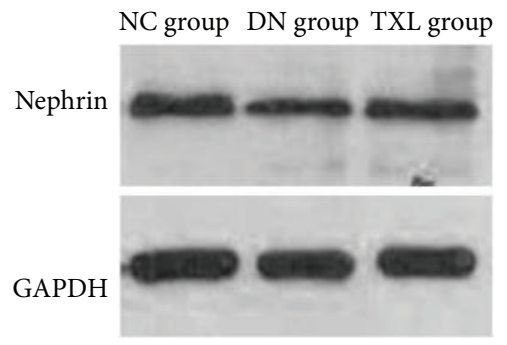

(a)
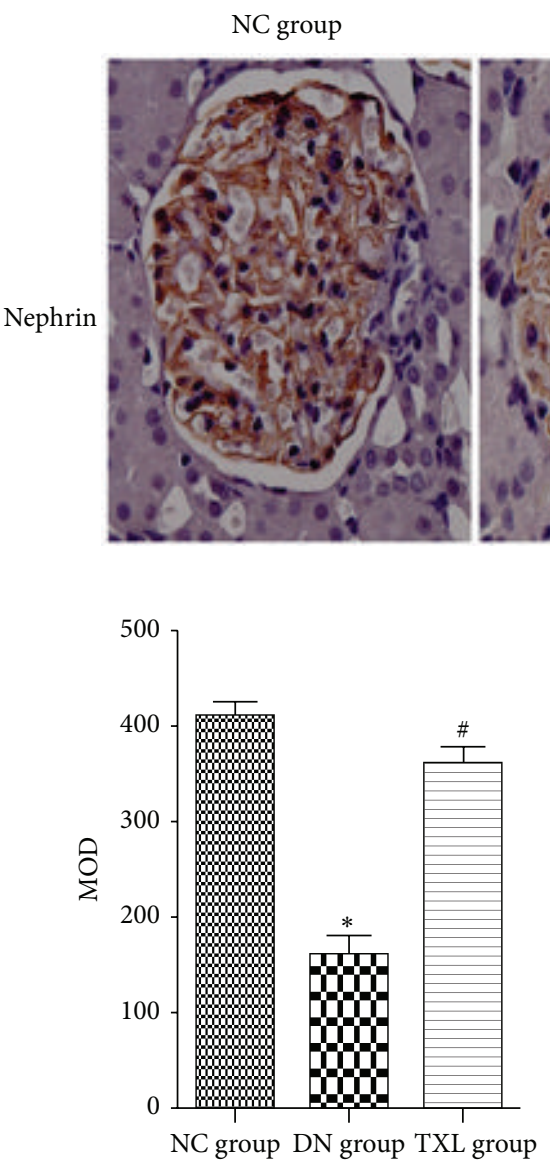

(d)

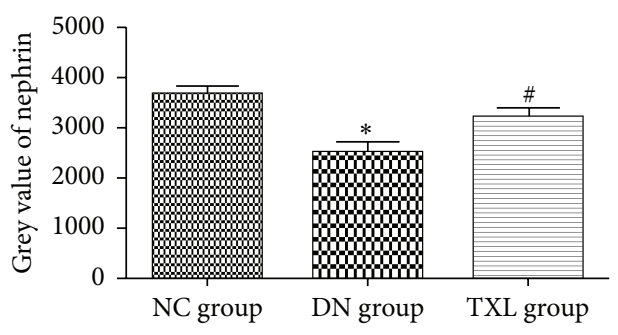

(b)
DN group

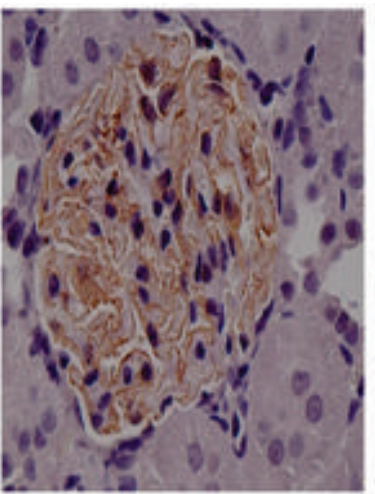

(c)

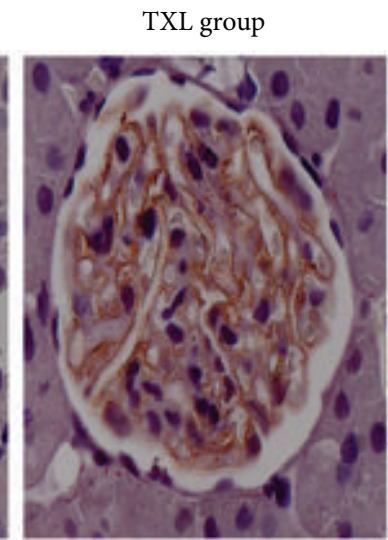

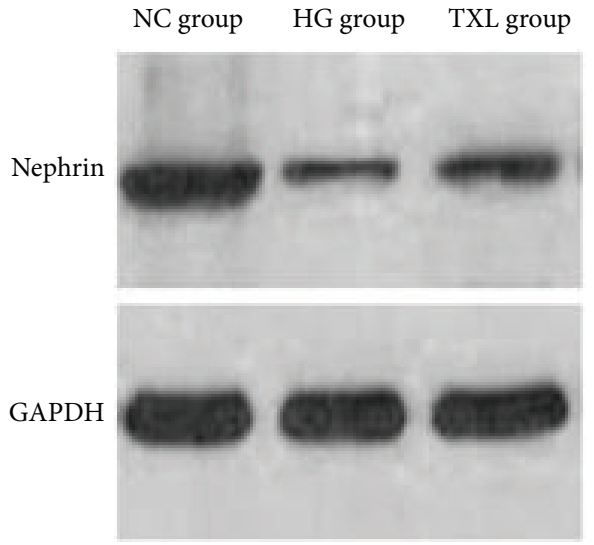

(e)

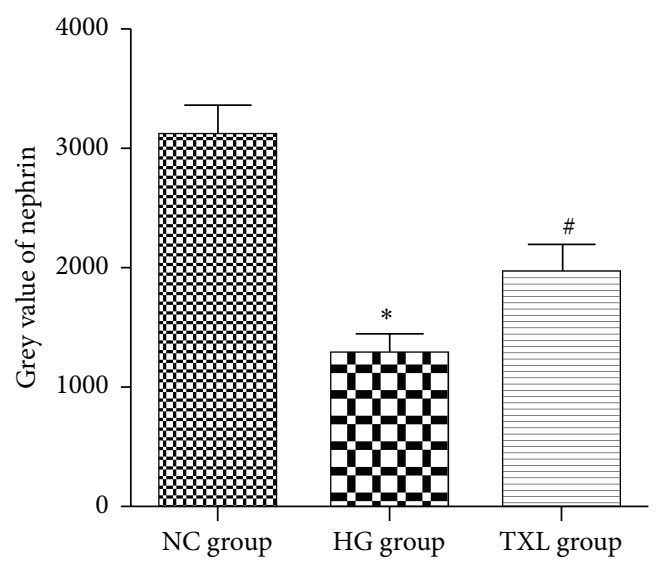

(f)

FIgure 2: Continued. 


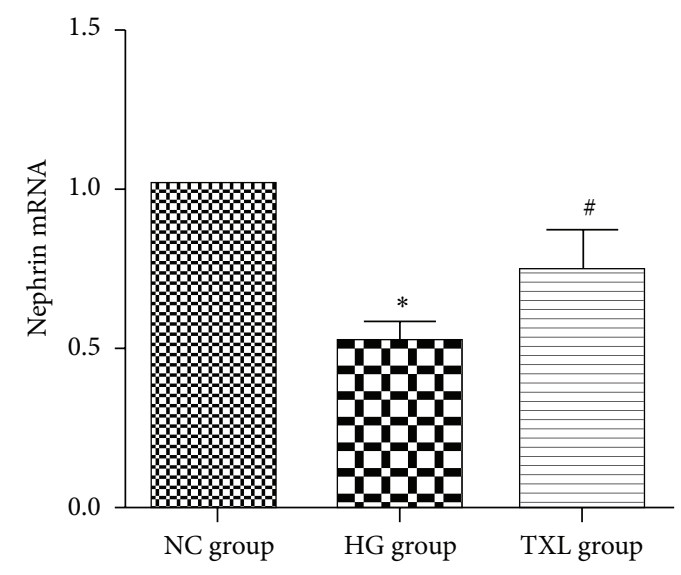

(g)

FIGURE 2: Effect of TXL on nephrin expression in diabetic rats and high glucose cultured podocyte. (a) Representative band of nephrin protein by western blot in rats. (b) Comparison of the grey value of nephrin protein in rats $(n=3)$. The result showed that nephrin protein was statistically significantly decreased in DN group compared with NC group $(P<0.05)$. Nephrin protein expression was significantly increased in TXL group compared with DN group $(P<0.05)$. (c) Representative immunohistochemical photograph for nephrin in vivo. Immunohistochemical results showed that nephrin protein expression was significantly decreased in DN group. (d) Comparison of mean optical density (MOD) of nephrin protein in rats. The result showed that nephrin protein was statistically significantly decreased in DN group compared with NC group $(P<0.05)$. Nephrin protein expression was significantly increased in TXL group compared with DN group $(P<0.05)$. (e) Representative band of nephrin protein by western blot in cultured podocyte. (f) Comparison of the grey value of nephrin protein in cultured podocyte $(n=3)$. The result showed that nephrin protein was statistically significantly decreased in HG group compared with NC group $(P<0.05)$. Nephrin protein expression was significantly increased in TXL group compared with HG group $(P<0.05)$. (g) Comparison of mRNA level of nephrin by RT-PCR in cultured podocyte $(n=3)$. The result showed that nephrin mRNA was statistically significantly decreased in HG group compared with NC group $(P<0.05)$. Nephrin mRNA expression was significantly increased in TXL group compared with HG group $(P<0.05) .{ }^{*} P<0.05$ versus NG. ${ }^{*} P<0.05$ versus $\mathrm{HG}$.

ICN1 expression and nuclear translocation. ICN1 protein expression, detected by western blot, was increased in HG group compared with NC group $(P<0.05)$. TXL markedly decreased ICN1 protein expression induced by HG $(P<$ 0.05). Moreover, high glucose significantly increased HES1 mRNA expression $(P<0.05)$. TXL markedly decreased HES1 mRNA expression induced by HG $(P<0.05)$ (Figure 3$)$.

3.4. TXL Decreased Snail Expression In Vivo and In Vitro. Snail, a DNA-binding molecule, is increased in injured podocyte. It has been demonstrated that snail directly decreased nephrin expression through binding the segment $\mathrm{Cl}$ of nephrin gene [25]. Moreover, HES1 can directly regulate snail expression in other cell lines [26]. Thus, snail acts as a bridge for decreased nephrin expression and activated notch pathway in podocyte in DN. Similarly, snail expression of renal cortex was detected by immunohistochemistry in our in vivo study. Our results showed that snail expression was increased in DN group compared with NC group. TXL decreased snail expression in diabetic rats. Next, the effect of TXL on snail was explored in cultured podocyte. Immunofluorescence results showed that snail weakly located in the cytoplasm of podocyte in NC group. In HG group, snail was transferred into the nucleus and its expression was increased. TXL inhibited snail expression and nuclear translocation. Moreover, snail protein and mRNA expression level, detected by western blot and RT-PCR, were significantly increased in HG group compared with NC group $(P<0.05)$.
TXL markedly inhibited snail protein and mRNA expression induced by HG $(P<0.05)$ (Figure 4$)$.

3.5. TXL Protected Podocyte from Injury In Vivo and In Vitro. Decreased nephrin expression has an intensive relationship with podocyte apoptosis and podocyte cytoskeleton change. The change of podocyte cytoskeleton can lead to podocyte foot process effacement and proteinuria. Herein, UAE, podocyte foot process, podocyte cytoskeleton, and podocyte apoptosis were detected in our in vivo and in vitro study. In our in vivo study, UAE was increased and podocyte foot process became fused and flattened in DN group. TXL significantly decreased UAE and alleviated podocyte foot effacement in diabetic rats. Our in vitro study showed that apoptotic cells were significantly increased in HG group compared with NC group $(P<0.05)$. Administration of TXL significantly decreased the number of apoptotic cells induced by high glucose $(P<0.05)$. In NC group, podocyte cytoskeleton was observed as parallel bundles of stress fibers. In HG group, intracellular actin stress fibers were abolished and placed by cortical actin web, resulting in a polygonal cellular shape. Moreover, TXL resumed intracellular actin stress fibers and maintained the normal cellular shape of high glucose cultured podocyte (Figure 5).

\section{Discussion}

Accumulate evidence has shown that podocyte loss and injury are an important mechanism of DN [2-4]. Nephrin is a 


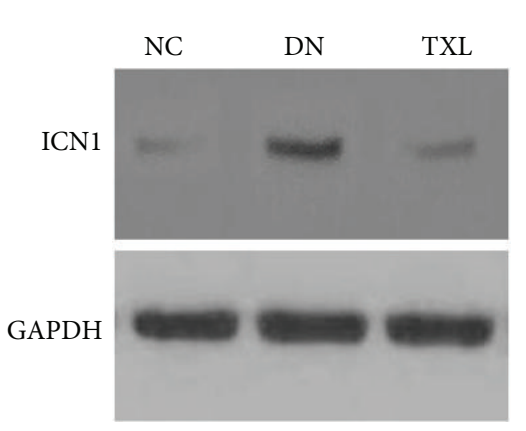

(a)

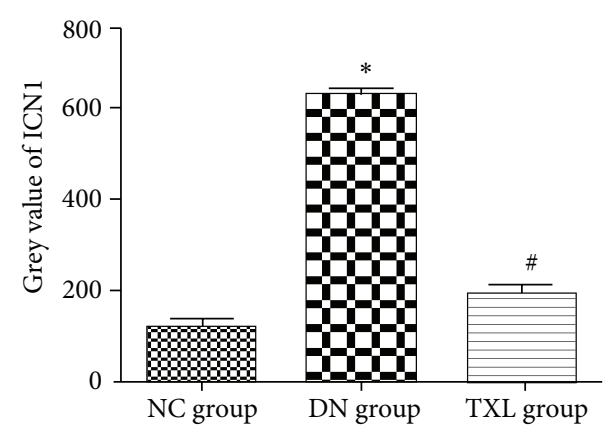

(b)

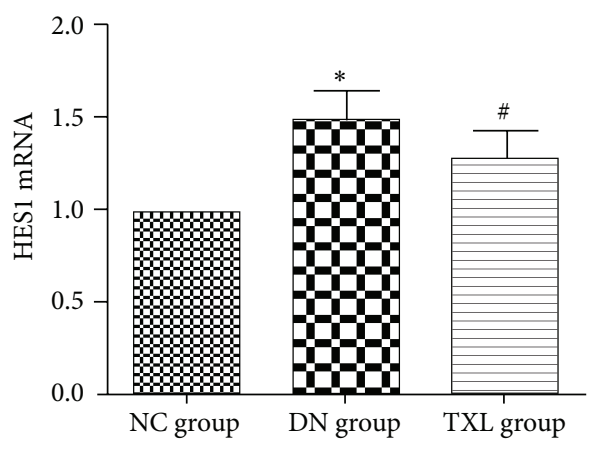

(c)

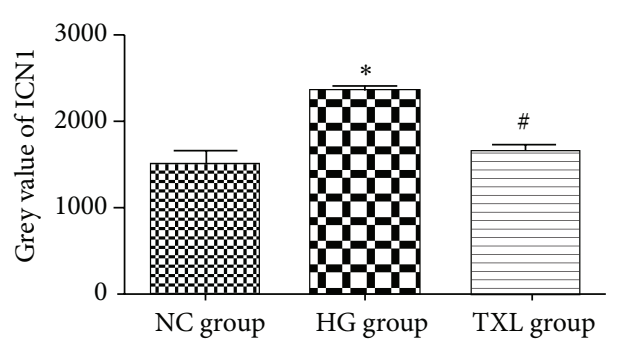

(f)

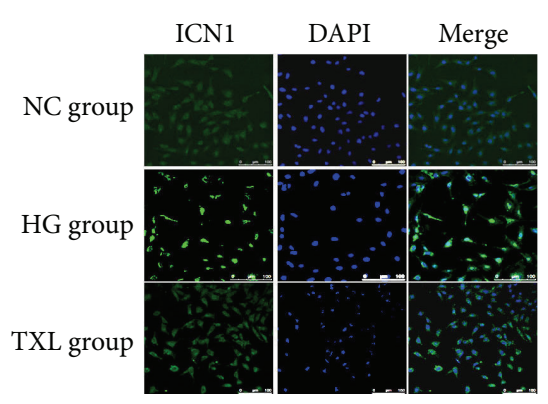

(d)

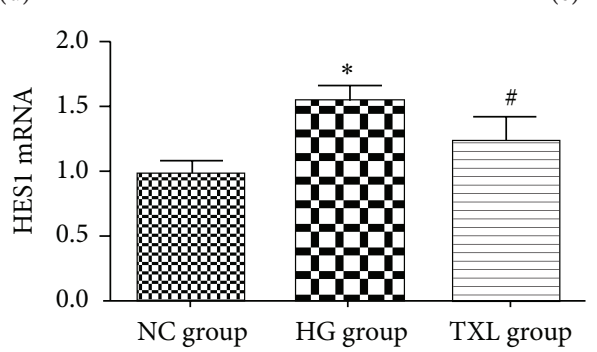

(g)

FIGURE 3: Effect of TXL on notch pathway in diabetic rats and high glucose cultured podocyte. (a) Representative band of ICN1 protein by western blot in rats. (b) Comparison of the grey value of ICN1 protein in rats $(n=3)$. The result showed that ICN1 protein was statistically significantly increased in DN group compared with NC group $(P<0.05)$. ICN1 protein expression was significantly decreased in TXL group compared with DN group $(P<0.05)$. (c) Comparison of mRNA level of HES1 by RT-PCR in rats $(n=3)$. The result showed that HES1 mRNA was statistically significantly increased in DN group compared with NC group $(P<0.05)$. HES1 mRNA expression was significantly decreased in TXL group compared with DN group $(P<0.05)$. (d) Representative photograph of ICN1 staining (green) and cell nucleus (DAPI blue). High glucose increased ICN1 expression and promoted ICN1 translocation from cytoplasm to nucleus, and TXL inhibited ICN1 expression and nuclear translocation. (e) Representative band of ICN1 protein by western blot in cultured podocyte. (f) Comparison of the grey value of ICN1 protein in cultured podocyte $(n=3)$. The result showed that ICN1 protein was statistically significantly increased in HG group compared with NC group $(P<0.05)$. ICN1 protein expression was significantly decreased in TXL group compared with HG group $(P<0.05)$. (g) Comparison of mRNA level of HES1 by RT-PCR in cultured podocyte $(n=3)$. The result showed that HES1 mRNA was statistically significantly increased in HG group compared with NC group $(P<0.05)$. HES1 mRNA expression was significantly decreased in TXL group compared with HG group $(P<0.05) .{ }^{*} P<0.05$ versus NG. ${ }^{\#} P<0.05$ versus HG.

signature molecule of podocyte [5]. It has been demonstrated that nephrin expression is significantly decreased in DN [69]. In accordance with previous studies, our study showed that nephrin expression was decreased in diabetic rats and in high glucose cultured podocyte. More importantly, TXL increased nephrin expression in vivo and in vitro.

Evidence is available that notch pathway is a common pathway of podocyte injury [10]. It has been demonstrated that the activation of notch pathway can downregulate nephrin expression in previous studies [11, 12]. Meanwhile, Gagliardini et al. demonstrate that persistent activation of notchl and snail signaling in podocyte contributes to decreased nephrin expression in DN [13]. In our study, ICN1, HES1, and snail expression were increased in diabetic rats and in high glucose cultured podocyte, indicating the activation of notch/snail pathway. Moreover, the administration of TXL significantly inhibited the activation of notch/snail pathway in vivo and in vitro. 


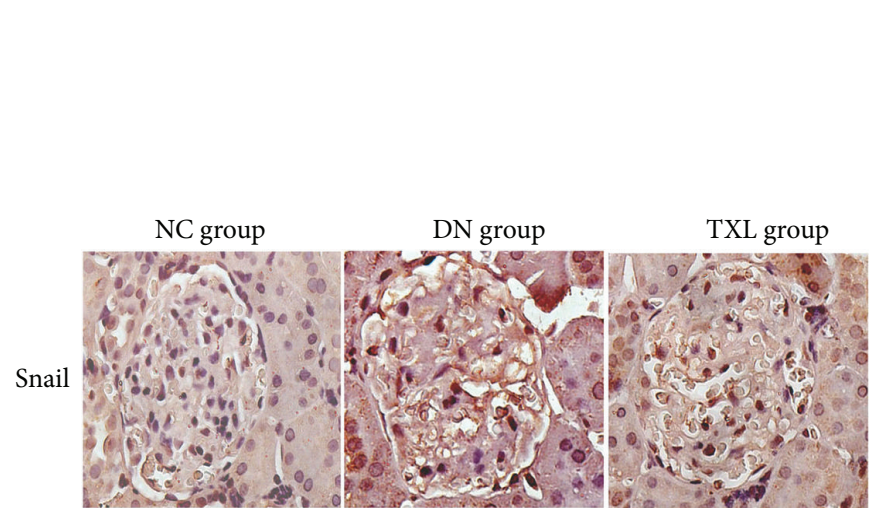

(a)

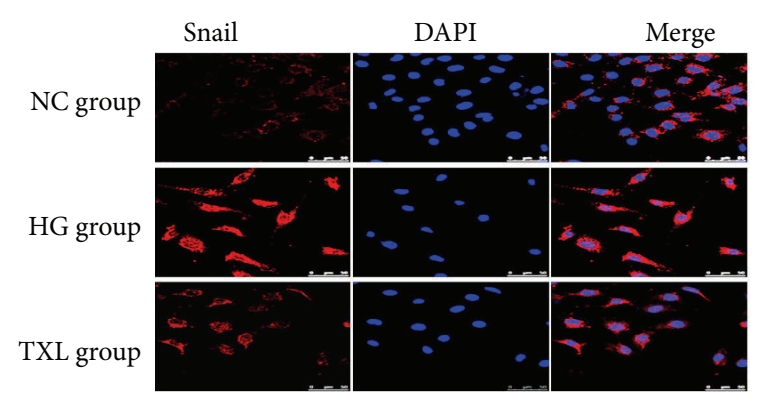

(c)

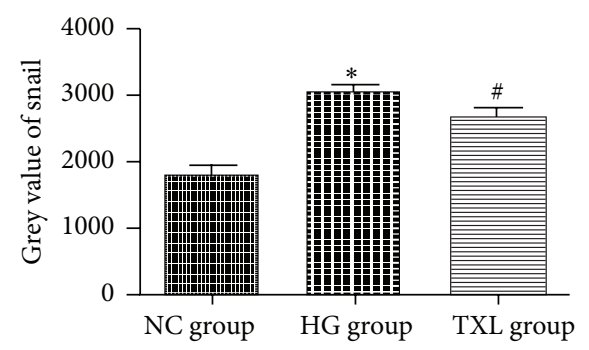

(e)

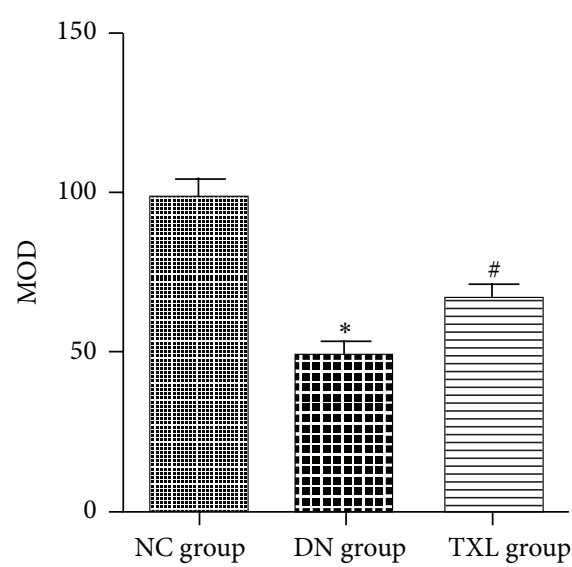

(b)

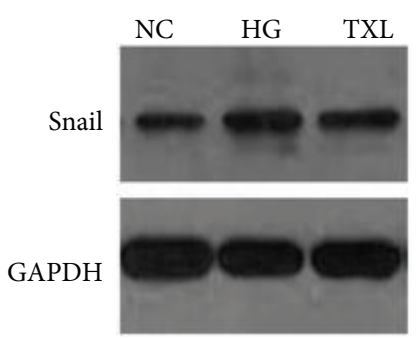

(d)

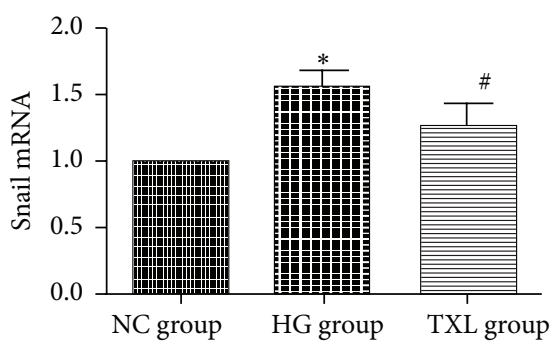

(f)

FIGURE 4: Effect of TXL on snail expression in diabetic rats and high glucose cultured podocyte. (a) Representative immunohistochemical photograph for snail in vivo. Immunohistochemical results showed that nephrin protein expression was significantly increased and translocated from cytoplasm to nucleus in DN group. TXL decreased snail protein expression and inhibited the translocation from cytoplasm to nucleus in diabetic rats. (b) Comparison of mean optical density (MOD) of snail protein in rats. The result showed that snail protein was statistically significantly decreased in DN group compared with NC group $(P<0.05)$. Snail protein expression was significantly increased in TXL group compared with DN group $(P<0.05)$. (c) Representative photograph of snail staining (red) and cell nucleus (DAPI blue). High glucose increased snail expression and promoted snail translocation from cytoplasm to nucleus, and TXL inhibited snail expression and nuclear translocation. (d) Representative band of snail protein by western blot in cultured podocyte. (e) Comparison of the grey value of snail protein in cultured podocyte $(n=3)$. The result showed that snail protein was statistically significantly increased in HG group compared with NC group $(P<0.05)$. Snail protein expression was significantly decreased in TXL group compared with HG group $(P<0.05)$. (f) Comparison of mRNA level of snail by RT-PCR in cultured podocyte $(n=3)$. The result showed that snail mRNA was statistically significantly increased in HG group compared with NC group $(P<0.05)$. Snail mRNA expression was significantly decreased in TXL group compared with HG group $(P<0.05) .{ }^{*} P<0.05$ versus NG. ${ }^{\#} P<0.05$ versus $\mathrm{HG}$.

As we know, UAE is increased and podocyte foot becomes effaceable in DN. Moreover, previous studies have demonstrated that decreased nephrin expression can lead to podocyte apoptosis and podocyte cytoskeleton change [27-29]. Our study also found that UAE was increased and podocyte foot became effaceable in diabetic rats, accompanied with decreased nephrin expression. Meanwhile, HG significantly increased the number of apoptotic cells and induced the change of cytoskeleton in cultured podocyte in our in vitro study. As TXL alleviated the downregulation of nephrin in podocyte, the effect of TXL on podocyte injury in DN was explored in our study. Our results showed that administration of TXL significantly decreased UAE and ameliorated podocyte foot effacement in diabetic rats. Moreover, 


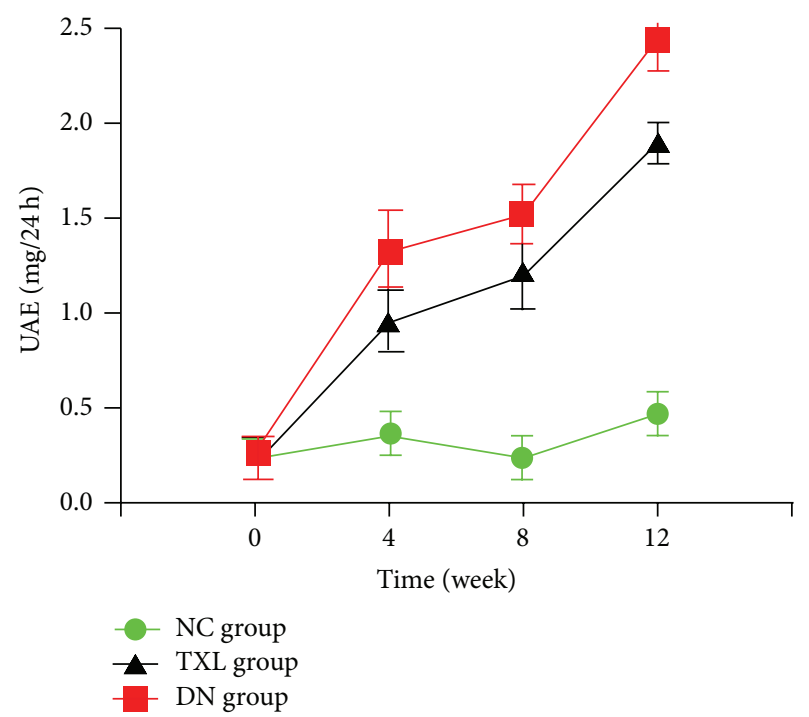

(a)

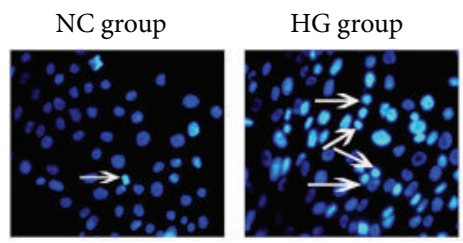

(c)

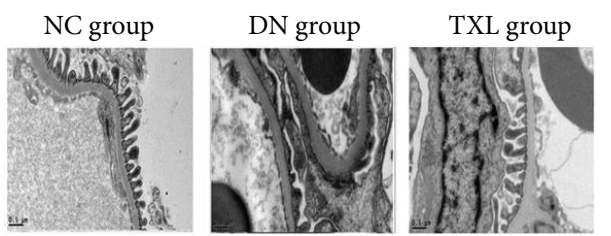

(b)
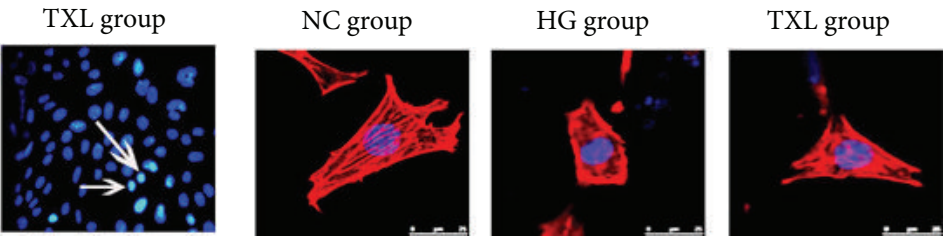

(d)

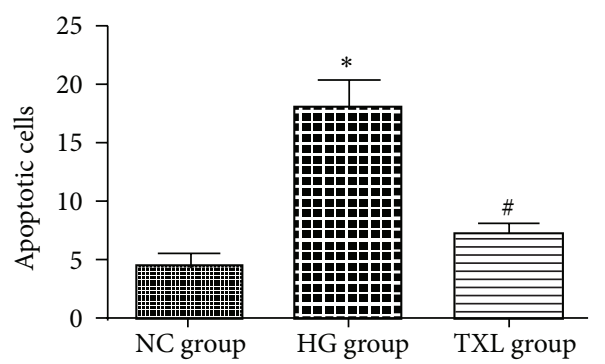

(e)

FIGURE 5: Effect of TXL on podocyte injury in diabetic rats and in high glucose cultured podocyte. (a) Comparison of UAE in three groups $(n=12)$. At the beginning of our study, UAE was not significantly different in three groups. At the end of 4,8 , and 12 weeks, UAE was increased in a time-dependent manner in DN group. Compared with DN group, UAE was decreased in TXL group at the end of 4, 8, and 12 weeks. (b) Representative photograph of podocyte foot process by electron microscopy. Compared with NC group, podocyte foot became fused and flattened in DN group. TXL significantly ameliorated podocyte foot effacement in diabetic rats. (c) Representative photograph of apoptotic cells by Hoechst 33258 staining (arrows indicate cell nuclear condensation). (d) Representative photograph of podocyte cytoskeleton by phalloidin staining. In NC group and MA group, podocyte cytoskeleton was normally arranged as long stress fiber-like bundles. In HG group, intracellular actin stress fibers were abolished and placed by cortical actin web, resulting in a polygonal cellular shape. (e) Comparison of apoptotic cells in three groups. The average number of apoptotic cells was assessed in 10 random fields per group. Compared with NC group, apoptotic cells were significantly increased in HG group $(P<0.05)$. Apoptotic cells were significantly decreased in TXL group compared with HG group $(P<0.05) .{ }^{*} P<0.05$ versus NG. ${ }^{*} P<0.05$ versus HG.

TXL significantly decreased the number of apoptotic cells and resumed abnormal cytoskeleton. Thus, we conclude that TXL has a protecting effect on podocyte injury in DN.

\section{Conclusion}

In conclusion, we speculated that TXL might have a pleiotropic effect on notch/snail pathway and nephrin expression both indirectly and directly, which may be a mechanism of protected effect on podocyte injury in DN. However, our study has some limitations and pitfalls. Although TXL has a pleiotropic effect on podocyte injury, the main therapeutic target of TXL is still unclear. Thus, further studies need to be performed.

\section{Conflict of Interests}

The authors declare that they have no conflict of interests regarding the publication of this paper. 


\section{Acknowledgment}

This work was supported by Grants from the Major National Basic Research Program of China (973 Program, no. 2012CB518602).

\section{References}

[1] H. Pavenstädt, W. Kriz, and M. Kretzler, "Cell biology of the glomerular podocyte," Physiological Reviews, vol. 83, no. 1, pp. 253-307, 2003.

[2] C. Dai, J. Yang, S. Bastacky, J. Xia, Y. Li, and Y. Liu, "Intravenous administration of hepatocyte growth factor gene ameliorates diabetic nephropathy in mice," Journal of the American Society of Nephrology, vol. 15, no. 10, pp. 2637-2647, 2004.

[3] M. Toyoda, B. Najafian, Y. Kim, M. L. Caramori, and M. Mauer, "Podocyte detachment and reduced glomerular capillary endothelial fenestration in human type 1 diabetic nephropathy," Diabetes, vol. 56, no. 8, pp. 2155-2160, 2007.

[4] M. Dalla Vestra, A. Masiero, A. M. Roiter, A. Saller, G. Crepaldi, and P. Fioretto, "Is podocyte injury relevant in diabetic nephropathy? Studies in patients with type 2 diabetes," Diabetes, vol. 52, no. 4, pp. 1031-1035, 2003.

[5] G. I. Welsh and M. A. Saleem, "Nephrin-signature molecule of the glomerular podocyte?" Journal of Pathology, vol. 220, no. 3, pp. 328-337, 2010.

[6] C. Li and H. M. Siragy, "High glucose induces podocyte injury via enhanced (Pro)renin receptor-Wnt- $\beta$-catenin-snail signaling pathway," PLoS ONE, vol. 9, no. 2, Article ID e89233, 2014.

[7] G. Li, Y. Li, S. Liu et al., "Gremlin aggravates hyperglycemiainduced podocyte injury by a TGF $\beta /$ smad dependent signaling pathway," Journal of Cellular Biochemistry, vol. 114, no. 9, pp. 2101-2113, 2013.

[8] S. Doublier, G. Salvidio, E. Lupia et al., "Nephrin expression is reduced in human diabetic nephropathy: evidence for a distinct role for glycated albumin and angiotensin II," Diabetes, vol. 52, no. 4, pp. 1023-1030, 2003.

[9] A. Benigni, E. Gagliardini, S. Tomasoni et al., "Selective impairment of gene expression and assembly of nephrin in human diabetic nephropathy," Kidney International, vol. 65, no. 6, pp. 2193-2200, 2004.

[10] L. Barisoni, "Notch signaling: a common pathway of injury in podocytopathies?" Journal of the American Society of Nephrology, vol. 19, no. 6, pp. 1045-1046, 2008.

[11] A. M. Waters, M. Y. J. Wu, T. Onay et al., "Ectopic Notch activation in developing podocytes causes glomerulosclerosis," Journal of the American Society of Nephrology, vol. 19, no. 6, pp. 1139-1157, 2008.

[12] T. Niranjan, B. Bielesz, A. Gruenwald et al., "The Notch pathway in podocytes plays a role in the development of glomerular disease," Nature Medicine, vol. 14, no. 3, pp. 290-298, 2008.

[13] E. Gagliardini, N. Perico, P. Rizzo et al., "Angiotensin II contributes to diabetic renal dysfunction in rodents and humans via notch1/snail pathway," American Journal of Pathology, vol. 183, no. 1, pp. 119-130, 2013.

[14] G. C. Xu, R. L. Gao, and Y. L. Wu, "Clinical study on tongxinluo capsule in treatment of patients with angina pectoris caused by coronary heart disease," Zhongguo Zhong Xi Yi Jie He Za Zhi, vol. 17, no. 7, pp. 414-416, 1997.
[15] G.-Q. Yuan, Y.-L. Wu, and Z.-H. Jia, "Experimental study on effect of Tongxinluo on nerve cell apoptosis after cerebral ischemia in middle cerebral arterial obstructive model rats," Zhongguo Zhong Xi Yi Jie He Za Zhi, vol. 27, no. 8, pp. 720-723, 2007.

[16] B. Wang, Q. Yang, W.-W. Bai et al., "Tongxinluo protects against pressure overload-induced heart failure in mice involving VEGF/Akt/eNOS pathway activation," PLoS ONE, vol. 9, no. 6, Article ID e98047, 2014.

[17] H. Cui, X. Li, N. Li et al., "Induction of autophagy by Tongxinluo through the MEK/ERK pathway protects human cardiac microvascular endothelial cells from hypoxia/reoxygenation injury," Journal of Cardiovascular Pharmacology, vol. 64, no. 2, pp. 180-190, 2014.

[18] Y. Liu, G. H. Tang, Y. H. Sun et al., “The protective role of Tongxinluo on blood-brain barrier after ischemia-reperfusion brain injury," Journal of Ethnopharmacology, vol. 148, no. 2, pp. 632-639, 2013.

[19] N. Mao, Y. Cheng, X.-L. Shi et al., "Ginsenoside Rg1 protects mouse podocytes from aldosterone-induced injury in vitro," Acta Pharmacologica Sinica, vol. 35, no. 4, pp. 513-522, 2014.

[20] M.-H. Zhang, J.-M. Fan, X.-S. Xie et al., "Ginsenoside-Rg1 protects podocytes from complement mediated injury," Journal of Ethnopharmacology, vol. 137, no. 1, pp. 99-107, 2011.

[21] J.-Y. Wang, Y.-B. Gao, N. Zhang et al., “Tongxinluo ameliorates renal structure and function by regulating miR-21-induced epithelial-to-mesenchymal transition in diabetic nephropathy," American Journal of Physiology-Renal Physiology, vol. 306, no. 5, pp. F486-F495, 2014.

[22] J. Q. Liang, K. Wu, Z. H. Jia et al., “Chinese medicine Tongxinluo modulates vascular endothelial function by inducing eNOS expression via the PI-3K/Akt/HIF-dependent signaling pathway," Journal of Ethnopharmacology, vol. 133, no. 2, pp. 517-523, 2011.

[23] Y. H. Shen, L. Zhang, Y. Gan et al., "Up-regulation of PTEN (phosphatase and tensin homolog deleted on chromosome ten) mediates p38 MAPK stress signal-induced inhibition of insulin signaling. A cross-talk between stress signaling and insulin signaling in resistin-treated human endothelial cells," The Journal of Biological Chemistry, vol. 281, no. 12, pp. 77277736, 2006.

[24] C. Sahlgren, M. V. Gustafsson, S. Jin, L. Poellinger, and U. Lendahl, "Notch signaling mediates hypoxia-induced tumor cell migration and invasion," Proceedings of the National Academy of Sciences of the United States of America, vol. 105, no. 17, pp. 6392-6397, 2008.

[25] I. Matsui, T. Ito, H. Kurihara, E. Imai, T. Ogihara, and M. Hori, "Snail, a transcriptional regulator, represses nephrin expression in glomerular epithelial cells of nephrotic rats," Laboratory Investigation, vol. 87, no. 3, pp. 273-283, 2007.

[26] S. Sumual, S. Saad, O. Tang et al., "Differential regulation of Snail by hypoxia and hyperglycemia in human proximal tubule cells," International Journal of Biochemistry and Cell Biology, vol. 42, no. 10, pp. 1689-1697, 2010.

[27] R. R. Foster, M. A. Saleem, P. W. Mathieson, D. O. Bates, and S. J. Harper, "Vascular endothelial growth factor and nephrin interact and reduce apoptosis in human podocytes," American Journal of Physiology-Renal Physiology, vol. 288, no. 1, pp. F48F57, 2005. 
[28] N. Jones, I. M. Blasutig, V. Eremina et al., "Nck adaptor proteins link nephrin to the actin cytoskeleton of kidney podocytes," Nature, vol. 440, no. 7085, pp. 818-823, 2006.

[29] R. Verma, I. Kovari, A. Soofi, D. Nihalani, K. Patrie, and L. B. Holzman, "Nephrin ectodomain engagement results in Src kinase activation, nephrin phosphorylation, Nck recruitment, and actin polymerization," The Journal of Clinical Investigation, vol. 116, no. 5, pp. 1346-1359, 2006. 


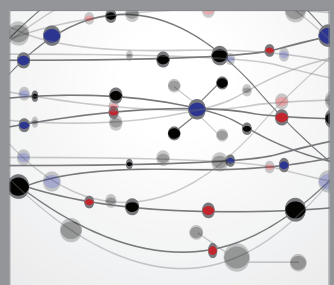

The Scientific World Journal
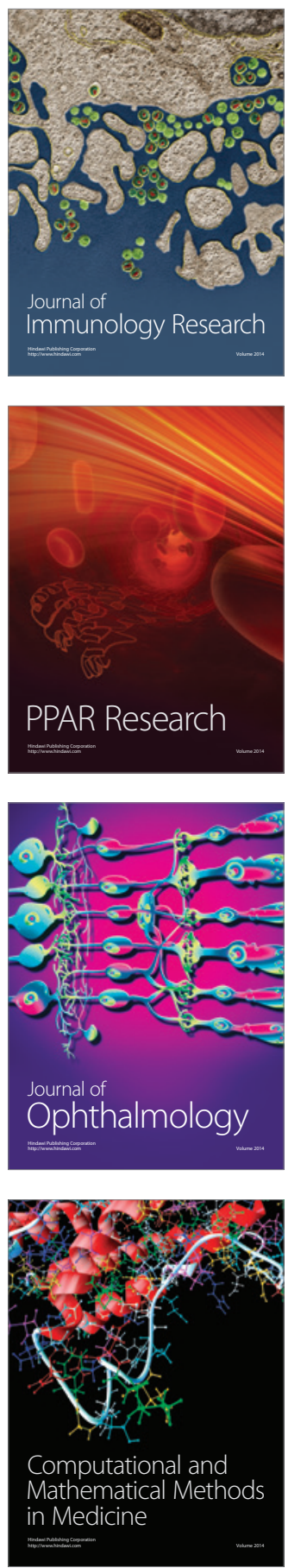

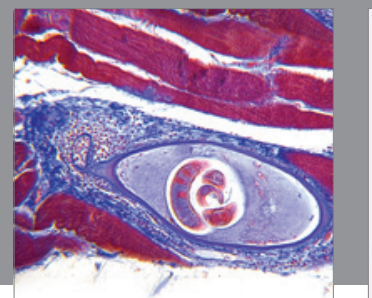

Gastroenterology

Research and Practice
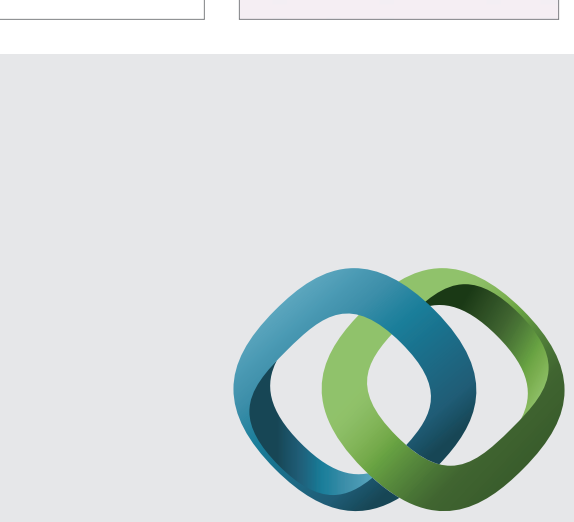

\section{Hindawi}

Submit your manuscripts at

http://www.hindawi.com
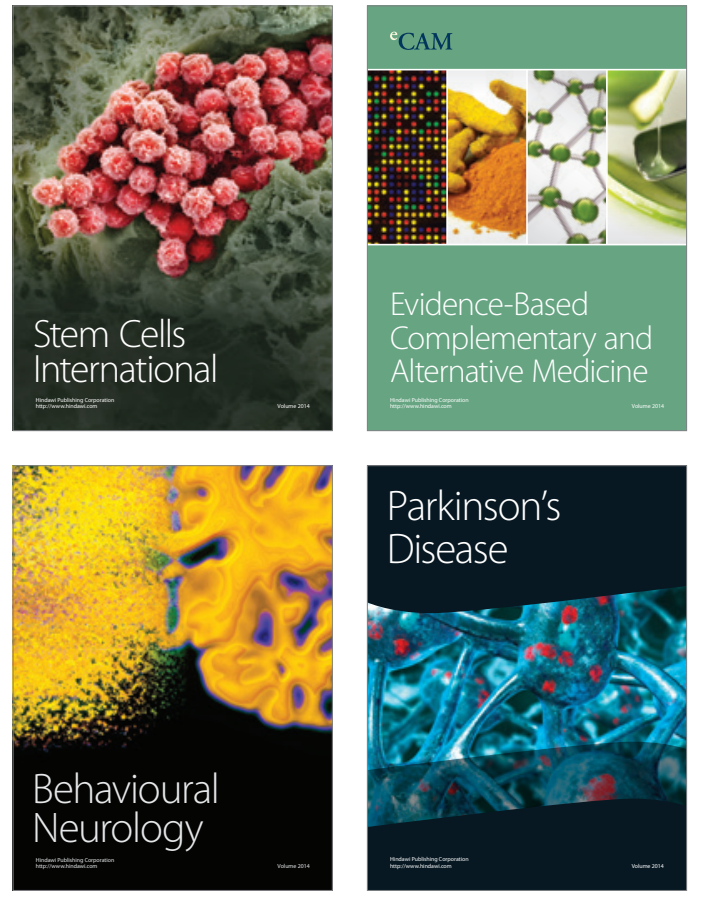
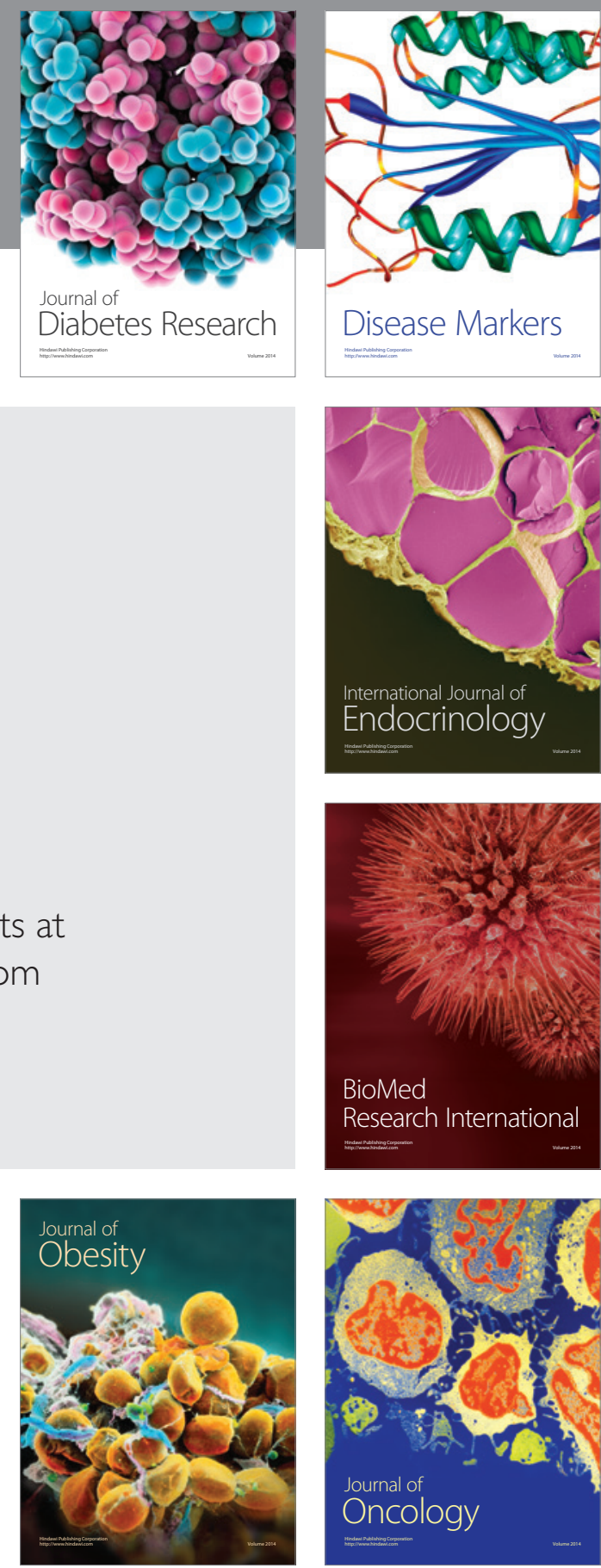

Disease Markers
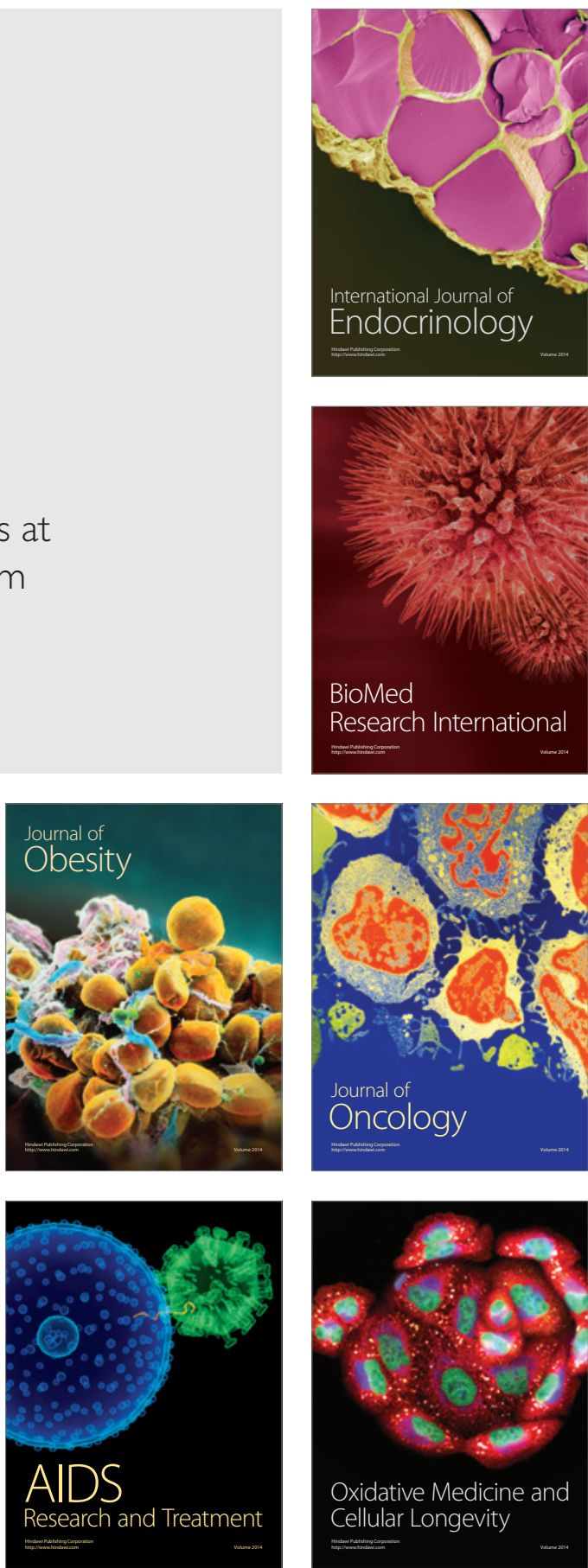